Review began 01/16/2022 Review ended 01/21/2022 Published 01/25/2022

๑) Copyright 2022

Gupta et al. This is an open access article distributed under the terms of the Creative Commons Attribution License CC-BY 4.0. which permits unrestricted use, distribution, and reproduction in any medium, provided the original author and source are credited.

\section{Dengue Encephalitis: A Case Series on a Rare Presentation of Dengue Fever}

\author{
Samiksha Gupta ${ }^{1}$, Gautam Jesrani ${ }^{1}$, Yuvraj S. Cheema ${ }^{1}$, Vivek Kumar ${ }^{1}$, Aman Garg ${ }^{1}$ \\ 1. Internal Medicine, Government Medical College and Hospital, Chandigarh, Chandigarh, IND
}

Corresponding author: Gautam Jesrani, jesranigautam@gmail.com

\begin{abstract}
Neurological manifestations like encephalitis, especially hemorrhagic encephalitis, are rarely described in dengue fever (DF), and the gamut may affect any part of the central or peripheral nervous system. Herein, we report two cases from Northern India, presenting with fever and altered sensorium, subsequently diagnosed with DF. Imaging studies revealed hemorrhagic encephalitis in both of them but one of them had a grave outcome, unfolding the fatal nature of the disease. The report enlightens DF as an unusual etiology of encephalitis and the importance of considering the infirmity as a differential in patients with neurological manifestations.
\end{abstract}

Categories: Internal Medicine, Neurology, Infectious Disease

Keywords: hemorrhagic encephalitis, seizures, dengue encephalitis, neurological manifestation, dengue fever

\section{Introduction}

Dengue virus (serotypes DEN-1, 2, 3, 4) is a Flavivirus, which is transmitted through the bite of an infected Aedes agypti mosquito, and as per the census of the World Health Organization (WHO), the number of dengue cases has increased to eight times in the last two decades [1]. DEN-2 and DEN-3 are the serotypes traditionally involved in neurological complications [2]. The virus can affect any part of the nervous system, including the peripheral nervous system (PNS) and ophthalmic structures, due to its neurotropic nature. The major identifiable risk factors contributing to neurological involvement are high-grade fever, thrombocytopenia, elevated hematocrit, and hepatic dysfunction [3].

\section{Case Presentation}

\section{Case 1}

A 37-year-old female homemaker, with no comorbidities, presented to the emergency department with altered mentation. There was a history of moderate grade, intermittent fever, and associated chills and rigor of five days' duration, along with a diffuse, dull, aching, continuous headache of three days' duration and three to four episodes of non-bilious vomiting prior to the presentation. There was no pain in the abdomen, diarrhea, or seizures. On examination, she was febrile $\left(102^{\circ} \mathrm{F}\right)$ but had a regular pulse rate of $102 \mathrm{beats} / \mathrm{min}$, blood pressure 100/80 mmHg, Glasgow coma scale (GCS) 8/15 (eye-opening: 2/4, verbal response: 3/5, motor response: $3 / 6$ ) and bilaterally reactive pupils. Meningeal signs were absent with bilateral flexor plantar response and no focal deficit. The rest of the systemic examination was normal.

She was then subjected to non-contrast computed tomography (NCCT) of the brain, which suggested diffuse cerebral edema (Figure 1). 


\section{Cureus}

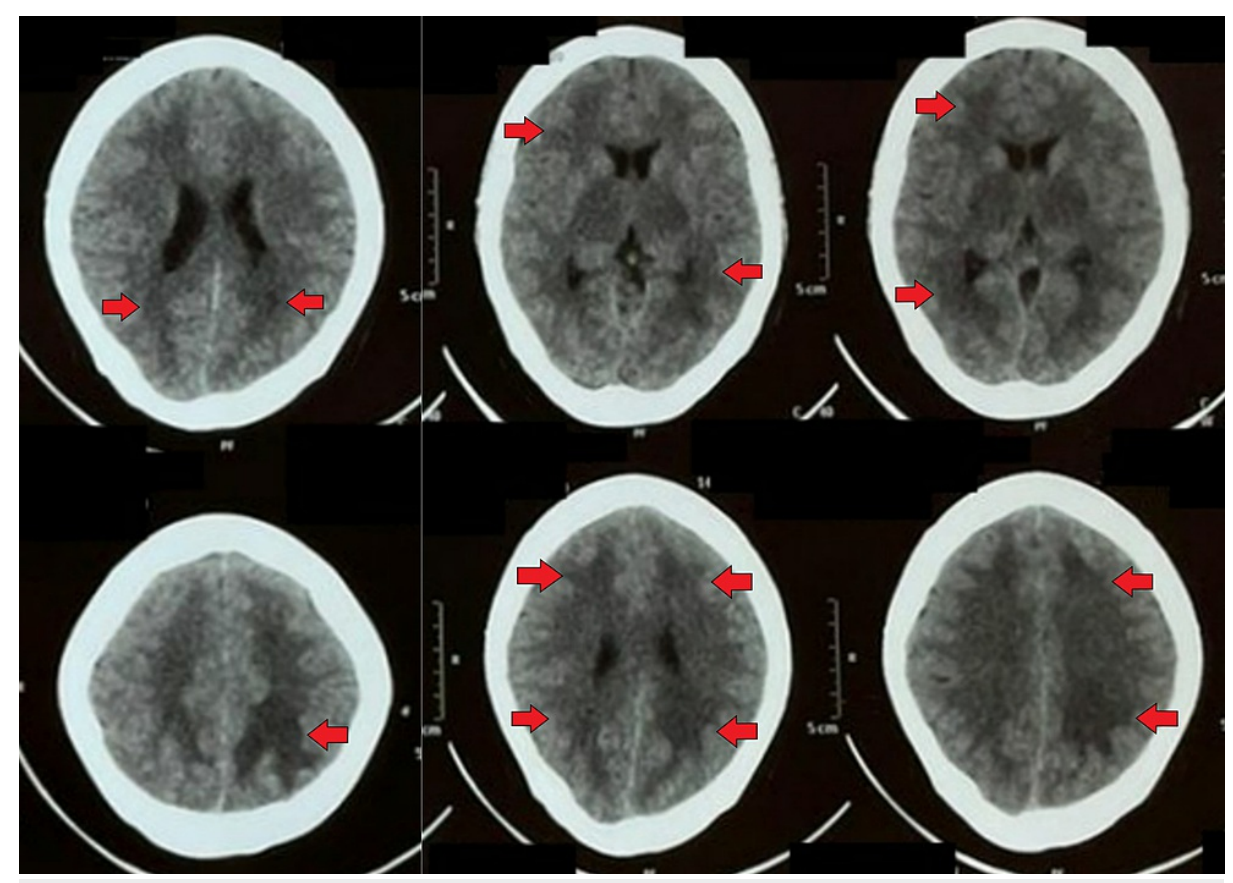

FIGURE 1: Non-contrast computed tomography of the brain demonstrating diffuse cerebral edema (red arrows)

On investigations, the patient had thrombocytopenia and transaminitis (Table 1). Cerebrospinal fluid (CSF) analysis had normal protein and glucose levels, no pleocytosis, and an absence of oligoclonal bands. On Gram staining and culture of the CSF, no organism was isolated and herpes simplex virus polymerase chain reaction (HSV PCR) was reported negative. She was managed on the lines of a presumptive diagnosis of infective encephalitis and started on intravenous ceftriaxone $2 \mathrm{gm}$ and dexamethasone $8 \mathrm{mg} 12$ hourly. 


\section{Cureus}

\begin{tabular}{|c|c|c|}
\hline INVESTIGATION & Value & Normal range \\
\hline Hemoglobin (g/dL) & 13.1 & $13-16$ \\
\hline Platelets $\left(\times 10^{9} / \mathrm{L}\right)$ & 76 & $150-400$ \\
\hline Total leukocyte count $\left(\times 10^{9} / \mathrm{L}\right)$ & 5.2 & $4-12$ \\
\hline Bilirubin (mg/dL) & 1.0 & $0.2-1$ \\
\hline ALP (IU/L) & 88 & $30-150$ \\
\hline SGOT (IU/L) & 409 & $10-40$ \\
\hline SGPT (IU/L) & 225 & $10-40$ \\
\hline Albumin (gm/dL) & 4.3 & $3.5-5.5$ \\
\hline Sodium (mmol/L) & 141 & $135-145$ \\
\hline Potassium (mmol/L) & 3.9 & $3.5-5.5$ \\
\hline Urea (mg/dL) & 31 & $15-40$ \\
\hline Creatinine (mg/dL) & 0.7 & $<1.3$ \\
\hline Dengue IgM serology & Positive & - \\
\hline Scrub typhus serology & Negative & - \\
\hline Malaria antigen & Negative & - \\
\hline Leptospira IgM serology & Negative & - \\
\hline Cytomegalovirus IgM serology & Negative & - \\
\hline Varicella-specific IgM serology & Negative & - \\
\hline Epstein-Barr virus IgM serology & Negative & - \\
\hline
\end{tabular}

\section{TABLE 1: Laboratory investigations of Case 1}

ALP, alkaline phosphatase; serum glutamic oxaloacetic transaminase, SGOT; serum glutamic pyruvic transaminase, SGPT; immunoglobulin, Ig

In view of thrombocytopenia and the ongoing endemic of tropical illnesses, the patient was investigated for malaria, dengue fever, scrub typhus, enteric fever, and leptospirosis. Later, contrast-enhanced magnetic resonance imaging (CEMRI) of the brain revealed hyperintensities in bilateral cerebral (frontal and temporal lobes) and cerebellar hemispheres, midbrain, and thalami (Figure 2). Susceptibility-weighted imaging suggested thalamic bleed (Figure 2) and all these features were suggestive of acute hemorrhagic necrotizing encephalitis. Angiography of the cerebral vessels was normal. Surprisingly, the patient came out to be Dengue NS-1 antigen positive by enzyme-linked immunoassay sorbent assay (ELISA), and from Day 3 onwards, she became afebrile with an improvement in GCS 13/15 (Eye-opening: 4/4, verbal response: 4/5, motor response: 5/6) and platelet count. Her antibiotic was stopped after three days and steroids were tapered but, unfortunately, she developed bilateral horizontal gaze palsy, dysarthria, and truncal ataxia. However, repeat NCCT head had no worsening or any new lesions. On the seventh day, the patient was discharged with a GCS of $15 / 15$ and resolving transaminitis. Follow-up at one month demonstrated minimal improvement in her residual symptoms. 


\section{Cureus}
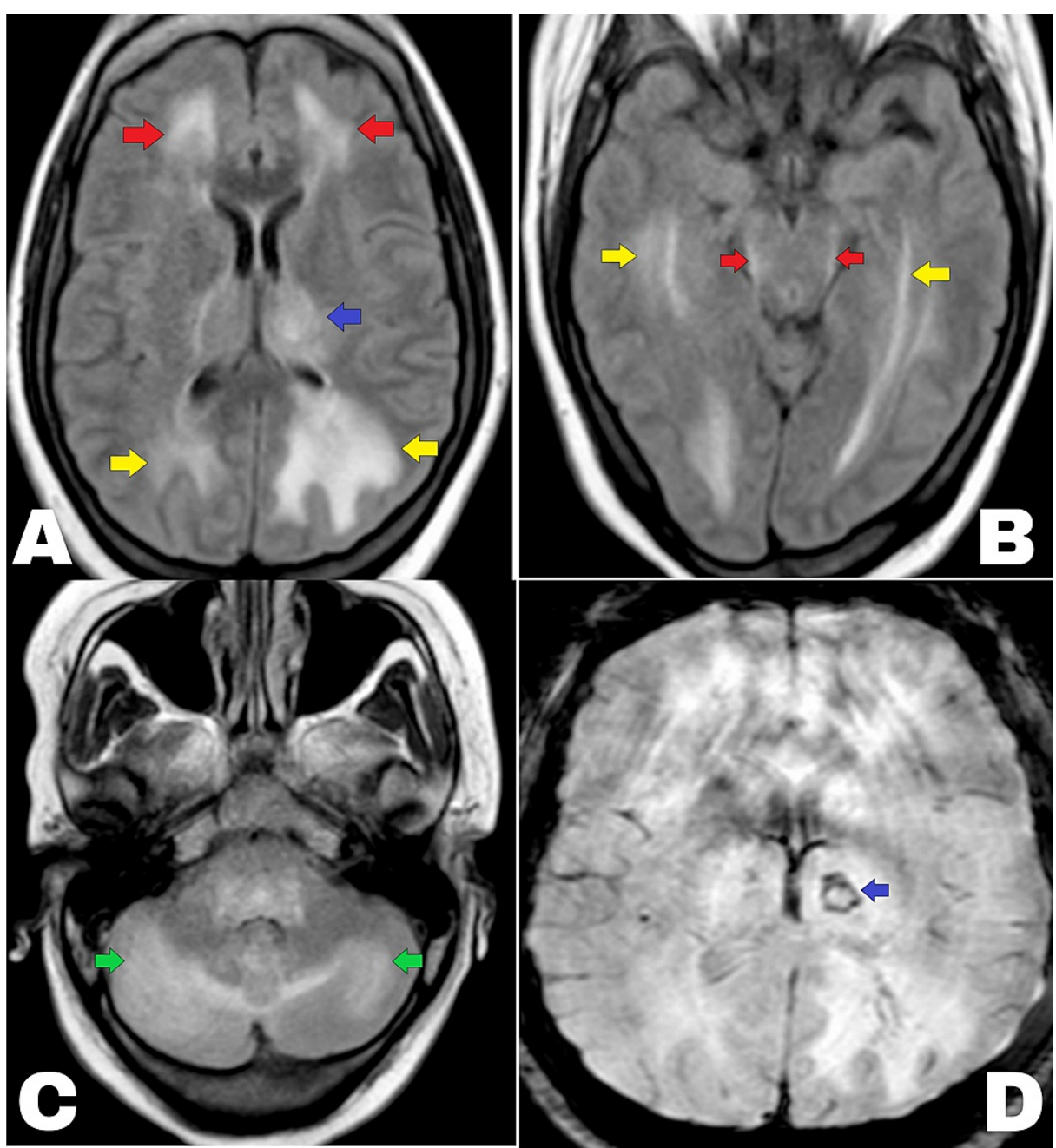

FIGURE 2: Magnetic resonance imaging (fluid-attenuated inversion recovery imaging) demonstrating hyperintensities in bilateral cerebral [frontal lobes (red arrow) and left thalamus (blue arrow) and occipital lobes (yellow arrow) in A], midbrain (red arrow) and temporal lobes (yellow arrow) in B, and cerebellar hemispheres (green arrow in C). Susceptibility-weighted imaging (D) suggested thalamic bleed (blue arrow).

\section{Case 2}

A 27-year-old male, with no history of addictions or co-morbidities, presented in poor sensorium, after three episodes of generalized tonic-clonic seizures in six hours' duration. There was a history of high-grade fever and associated chills and rigor for four days and persistent non-bilious vomiting for two days. No prior history of concurrent headache, motor weakness, or similar past episodes was documented. On presentation, he was febrile $\left(103^{\circ} \mathrm{F}\right)$ and had a pulse rate of 115 beats/min, blood pressure 98/70 $\mathrm{mmHg}$, GCS 10/15 (Eyeopening: $4 / 4$, verbal response: $2 / 5$, motor response: $4 / 6$ ), bilaterally reactive pupils, and absent meningeal signs. The other system examinations were unremarkable. So, he was started on anti-epileptics and an intravenous antibiotic (ceftriaxone 2 gm twice a day) initially.

His first NCCT brain indicated ill-defined hypodensity in bilateral thalami, bilateral cerebellar hemisphere, and medulla (Figure 3). Also, investigations suggested thrombocytopenia and deranged hepatic enzymes (Table 2). 


\section{Cureus}

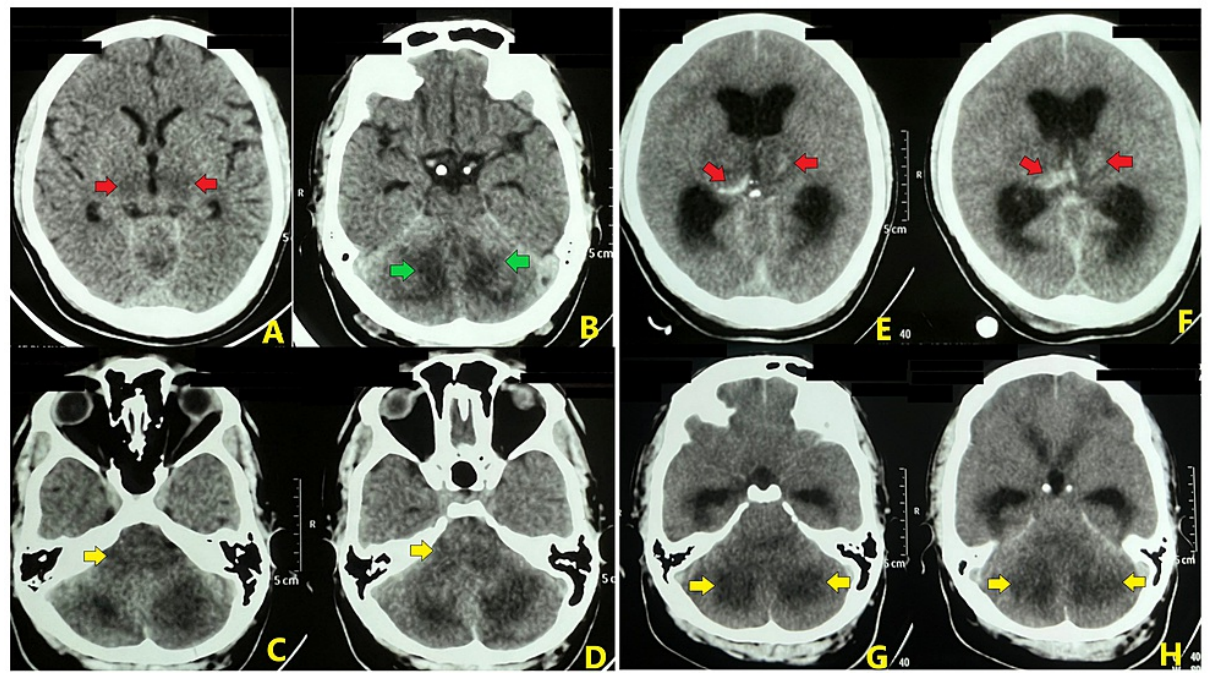

FIGURE 3: Non-contrast computed tomography of the brain demonstrating hypodensity in bilateral thalami (red arrow in A), bilateral cerebellar hemisphere (green arrow in B), and medulla (yellow arrow in C and D). Next computed tomography demonstrated intrathalamic hemorrhage (red arrow in $E$ and F) with diffuse cerebral edema hydrocephalus and diffuse hypodensities in the cerebellar hemisphere (yellow arrow in $\mathbf{G}$ and $\mathrm{H}$ ). 


\section{Cureus}

\begin{tabular}{|c|c|c|}
\hline INVESTIGATION & Value & Normal range \\
\hline Hemoglobin (g/dL) & 15.1 & $13-16$ \\
\hline Platelets $\left(\times 10^{9} / \mathrm{L}\right)$ & 15 & $150-400$ \\
\hline Total leukocyte count $\left(\times 10^{9} / \mathrm{L}\right)$ & 4.2 & $4-12$ \\
\hline Bilirubin (mg/dL) & 0.9 & $0.2-1$ \\
\hline ALP (IU/L) & 70 & $30-150$ \\
\hline SGOT (IU/L) & 1392 & $10-40$ \\
\hline SGPT (IU/L) & 813 & $10-40$ \\
\hline Albumin (gm/dL) & 3.9 & $3.5-5.5$ \\
\hline Sodium (mmol/L) & 137 & 135-145 \\
\hline Potassium (mmol/L) & 3.6 & $3.5-5.5$ \\
\hline Urea (mg/dL) & 28 & $15-40$ \\
\hline Creatinine (mg/dL) & 0.8 & $<1.3$ \\
\hline Hepatitis A serology & Negative & - \\
\hline Hepatitis E serology & Negative & - \\
\hline Hepatitis B serology & Negative & - \\
\hline Hepatitis C serology & Negative & - \\
\hline Dengue IgM serology & Positive & - \\
\hline Scrub typhus serology & Negative & - \\
\hline Malaria antigen & Negative & - \\
\hline Leptospira IgM serology & Negative & - \\
\hline Cytomegalovirus IgM serology & Negative & - \\
\hline Varicella-specific IgM serology & Negative & - \\
\hline Epstein-Barr virus IgM serology & Negative & - \\
\hline
\end{tabular}

\section{TABLE 2: Laboratory investigations of Case 2}

ALP, alkaline phosphatase; serum glutamic oxaloacetic transaminase, SGOT; serum glutamic pyruvic transaminase, SGPT; immunoglobulin, Ig

CSF analysis was deferred due to thrombocytopenia, and single donor platelets were transfused for the same. The patient had no seizure thereafter and platelet count improved to $56,000 \times 10^{9} / \mathrm{L}$ on the next day, so a lumbar puncture was performed. CSF analysis revealed normal protein and glucose levels, no pleocytosis, and an absence of oligoclonal bands. HSV PCR was reported negative, and serology for toxoplasma was inconclusive in CSF. Since there was no improvement in sensorium, CEMRI brain was planned, but his GCS deteriorated to 4/15 with bilaterally dilated pupils on the next day. The patient was intubated immediately and shifted to the intensive care unit. Repeat NCCT brain was done, which revealed diffuse cerebral and cerebellar edema, with compression of the fourth ventricle causing hydrocephalus and diffuse hypodensity in bilateral thalami and cerebellar hemisphere, with intrathalamic hemorrhage (Figure 3). Anti-edema measures were started [head end elevation, intravenous mannitol ( $0.5 \mathrm{mg} / \mathrm{kg}, 8 \mathrm{hourly})$, and dexamethasone ( $4 \mathrm{mg} 8$ hourly)]. However, the patient eventually succumbed to the illness four hours after the intubation. Post-obituary reports demonstrated positive Dengue NS-1 antigen by ELISA, negative serology for hepatitis A, E, B, and C, malaria, scrub typhus, typhoid fever, and leptospirosis. CSF culture did not reveal the growth of any organism and Indian ink preparation for cryptococcus was negative.

\section{Discussion}

As per the WHO 2009 classification, dengue encephalitis is categorized as severe dengue. Central nervous 
system (CNS) involvement (ischemic/hemorrhagic stroke, encephalitis, acute disseminated encephalomyelitis, and transverse myelitis), PNS manifestations (long thoracic nerve palsy, abducens nerve palsy, facial palsy, brachial neuritis, myositis, hypokalaemic paralysis, and Guillain-Barre syndrome), and ophthalmic complications (maculopathy, optic neuropathy, and subconjunctival and vitreous hemorrhage) have been described in the context of DF [4-9].

Encephalitis is inflammation of the brain parenchyma, often due to viral infection. Dengue virus was earlier considered non-neurotropic, but recent observations have proven this wrong. Direct viral invasion in the CNS, possibly as a result of the blood brain barrier disruption, is the proposed pathogenesis for neural involvement. Additionally, autoimmune reactions and metabolic variations have also been established, which further worsen neurological infirmity [10-11].

Three to seven days is the usual interval between the onset of neurological symptoms and systemic features of DF. Headache, fever, altered sensorium, seizures, and focal neurological deficit, in the absence of any metabolic abnormalities, are the typical features of encephalitis. Thrombocytopenia is depicted in the hemogram, and CSF analysis reveals pleocytosis with viral growth on culture. Still, CSF with normal cellularity can be seen in $75 \%$ of cases of dengue encephalitis [12].

The majority of the patients with dengue encephalitis have normal findings on neuroimaging [13]. MRI is preferred over CT head, although the findings are often non-specific [13]. The commonly affected sites of CNS are the thalamus and basal ganglia, followed by the cerebral cortex and cerebellar hemispheres. Findings such as focal hemorrhage, patchy areas of diffusion restriction, and post-contrast enhancement have also been defined.

Adequate hydration with intravenous fluids, antipyretics for fever, seizures control with anti-epileptic drugs, and transfusion of blood products (if required) forms the fundamental treatment in DF. Raised intracranial pressure can be treated with head elevation, mannitol, and steroids, with regular scrutinization of consciousness level. Our patients were managed similarly; nevertheless, the patient in Case 2 succumbed to his illness. The prognosis in dengue encephalitis is good, although mortality can range up to 3.7\% [14].

\section{Conclusions}

Dengue encephalitis should be kept as a differential in patients with a short history of fever and altered sensorium in countries where the DF is endemic, especially in the post-monsoon season. Thrombocytopenia is generally a clue for tropical illnesses like dengue, malaria, and scrub typhus and can be used as a basis for the evaluation of these infirmities. Also, physicians should have a high index of clinical suspicion since the prognosis is good if managed on time.

\section{Additional Information \\ Disclosures}

Human subjects: Consent was obtained or waived by all participants in this study. Conflicts of interest: In compliance with the ICMJE uniform disclosure form, all authors declare the following: Payment/services info: All authors have declared that no financial support was received from any organization for the submitted work. Financial relationships: All authors have declared that they have no financial relationships at present or within the previous three years with any organizations that might have an interest in the submitted work. Other relationships: All authors have declared that there are no other relationships or activities that could appear to have influenced the submitted work.

\section{References}

1. World Health Organization. Dengue and severe dengue. (2021). Accessed: October 22, 2021: https://www.who.int/news-room/fact-sheets/detail/dengue-and-severe-dengue .

2. Soares CN, Cabral-Castro MJ, Peralta JM, Freitas MR, Puccioni-Sohler M: Meningitis determined by oligosymptomatic dengue virus type 3 infection: report of a case. Int J Infect Dis. 2010, 14:e150-2. 10.1016/j.ijid.2009.03.016

3. Sahu R, Verma R, Jain A, et al.: Neurologic complications in dengue virus infection: a prospective cohort study. Neurology. 2014, 83:1601-9. 10.1212/WNL.0000000000000935

4. Myint KS, Gibbons RV, Perng GC, Solomon T: Unravelling the neuropathogenesis of Japanese encephalitis . Trans R Soc Trop Med Hyg. 2007, 101:955-6. 10.1016/j.trstmh.2007.04.004

5. Garg RK, Rizvi I, Ingole R, Jain A, Malhotra HS, Kumar N, Batra D: Cortical laminar necrosis in dengue encephalitis-a case report. BMC Neurol. 2017, 17:79. 10.1186/s12883-017-0855-9

6. Siriyakorn N, Insiripong S: Fatal rhabdomyolysis in dengue hemorrhagic fever: a case report . Southeast Asian J Trop Med Public Health. 2015, 46:149-52.

7. Maurya PK, Kulshreshtha D, Singh AK, Thacker AK: Rapidly resolving weakness related to hypokalemia in patients infected with dengue virus. J Clin Neuromuscul Dis. 2016, 18:72-8. 10.1097/CND.0000000000000140

8. Umapathi T, Lim CS, Ooi EE, et al.: Asymptomatic dengue infection may trigger Guillain-Barré syndrome . J Peripher Nerv Syst. 2016, 21:375-7. 10.1111/jns.12190

9. Yip VC, Sanjay S, Koh YT: Ophthalmic complications of dengue fever: a systematic review . Ophthalmol 


\section{Cureus}

Ther. 2012, 1:2. 10.1007/s40123-012-0002-z

10. Domingues RB, Kuster GW, Onuki-Castro FL, Souza VA, Levi JE, Pannuti CS: Involvement of the central nervous system in patients with dengue virus infection. J Neurol Sci. 2008, 267:36-40.

10.1016/j.jns.2007.09.040

11. Basu A, Chaturvedi UC: Vascular endothelium: the battlefield of dengue viruses. FEMS Immunol Med Microbiol. 2008, 53:287-99. 10.1111/j.1574-695X.2008.00420.x

12. Soares CN, Cabral-Castro MJ, Peralta JM, de Freitas MR, Zalis M, Puccioni-Sohler M: Review of the etiologies of viral meningitis and encephalitis in a dengue endemic region. J Neurol Sci. 2011, 303:75-9. 10.1016/j.jns.2011.01.012

13. Madi D, Achappa B, Ramapuram JT, Chowta N, Laxman M, Mahalingam S: Dengue encephalitis-a rare manifestation of dengue fever. Asian Pac J Trop Biomed. 2014, 4:S70-2. 10.12980/APJTB.4.2014C1006

14. Araújo F, Nogueira R, Araújo Mde S, et al.: Dengue in patients with central nervous system manifestations, Brazil. Emerg Infect Dis. 2012, 18:677-9. 10.3201/eid1804.111552 